\title{
Radiation from Isolated Spectral Lines with Combined Doppler and Lorentz Broadening*
}

\author{
S. S. Penner and R. W. Kavanagh \\ Guggenheim Jet Propulsion Center, California Institute of Technology, Pasadena, California
}

(Received September 15, 1952)

\begin{abstract}
Methods for the calculation of spectral absorption coefficients for combined Doppler and Lorentz broadening are summarized. The "curves of growth" have been extended to cover the ranges of parameters which arise in spectroscopic studies on flames.
\end{abstract}

\section{BASIC RELATIONS}

$\mathrm{F}^{\mathrm{o}}$ OR combined Doppler and Lorentz broadening it is well known ${ }^{1}$ that the spectral absorption coefficient at the wave number $\omega, P(\omega)$, is given, in adequate approximation, by the relation

$P(\omega)=P^{\prime}(a / \pi) \int_{-\infty}^{+\infty}\left[a^{2}+(\xi-y)^{2}\right]^{-1}\left[\exp \left(-y^{2}\right)\right] d y$

where

$$
\begin{aligned}
P^{\prime} & =\left(S / \omega_{0}\right)\left(m c^{2} / 2 \pi k T\right)^{\frac{1}{2}}, \\
a & =\left[\left(b_{N}+b_{C}\right) / \omega_{0}\right]\left(m c^{2} / 2 k T\right)^{\frac{1}{2}}=\left(b_{N}+b_{C}\right)(\ln 2)^{\frac{1}{2}} / b_{D},
\end{aligned}
$$

and

$$
\xi=\left[\left(\omega-\omega_{0}\right) / \omega_{0}\right]\left(m c^{2} / 2 k T\right)^{\frac{1}{2}}=\left[\left(\omega-\omega_{0}\right) / b_{D}\right](\ln 2)^{\frac{1}{2}} .
$$

Thus $P^{\prime}$ represents the maximum value of the spectral absorption coefficient if only Doppler broadening occurred; $b_{N}, b_{C}$, and $b_{D}$ denote, respectively, the natural half-width, $\uparrow$ the half-width resulting from collision (Lorentz) broadening, and the half-width produced by Doppler broadening; $S$ is the integrated intensity of the spectral line under study whose center lies at $\omega_{0} ; m$ is the mass of the radiating molecule; $k$ equals the Boltzmann constant; $T$ represents the absolute temperature.

The total radiant intensity $A$ emitted by a spectral line is

$$
A=\int_{\Delta \omega} R^{0}(\omega)\{1-\exp [-P(\omega) X]\} d \omega
$$

where $X$ represents the optical density of the emitter (for example, in $\mathrm{cm}$-atmos). The integration interval $\Delta \omega$ in Eq. (2) can be extended from $-\infty$ to $+\infty$, with the line center chosen as origin, for isolated spectral lines, without appreciable error. In Eq. (2) the quantity $R^{0}(\omega)$ denotes the intensity of radiation emitted by a blackbody in the wave-number interval between $\omega$ and $\omega+d \omega$ into a solid angle of $2 \pi$ steradians per unit area per unit time. The quantity $R^{0}(\omega)$ can be set equal to

\footnotetext{
* Supported by the U. S. Office of Naval Research.

1 See, for example, A. C. G. Mitchell and M. W. Zemansky, Resonance Radiation and Excited Atoms (Cambridge University Press, Cambridge, 1934), Appendix I.

$\dagger$ The term half-width is used to denote one-half of the wave number interval for which $P(\omega) \geqslant \frac{1}{2} P^{\prime}$. A consistent set of units would involve $P$ in $\mathrm{cm}^{-1}$-atoms ${ }^{-1}, S$ in $\mathrm{cm}^{-2}$-atoms ${ }^{-1}$, and the half-widths in $\mathrm{cm}^{-1}$.
}

$R^{0}\left(\omega_{0}\right) \equiv R^{0}$ without sensible error. Thus the problem of evaluation of intensities emitted by isolated spectral lines reduces to the calculation of

$$
A / R^{0}=\int_{-\infty}^{+\infty}\{1-\exp [-P(\omega) X]\} d \omega,
$$

where $P(\omega)$ is given by Eq. (1). The spectral absorption coefficients must, of course, satisfy the relation

$$
\int_{-\infty}^{+\infty} P(\omega) d \omega=S
$$

Before proceeding with the general problem it appears desirable to review briefly several special cases.

\section{A. Pure Doppler Broadening}

For pure Doppler broadening $a=0$ and Eq. (1) becomes $^{2}$

Furthermore ${ }^{2,3}$

$$
P(\omega)=P^{\prime} \exp \left(-\xi^{2}\right) .
$$

$$
A / R^{0}=(S X) \sum_{n=0}^{\infty}\left[(n+1)^{\frac{1}{2}}(n+1) !\right]^{-1}\left(-P^{\prime} X\right)^{n} .
$$

Numerical values for $A / R^{0} S X$ have been given by Ladenburg $^{3}$ for $0.10 \leqslant P^{\prime} X \leqslant 1000$. For $0 \leqslant P^{\prime} X \leqslant 30$, Eq. (5) may be replaced by the following approximation

$$
A / R^{0}=S X \exp \left[-\frac{1}{2}\left(P^{\prime} X\right)^{\frac{1}{2}}\right] \text {. }
$$

For very large values of $P^{\prime} X$ the following asymptotic expansion for $A / R^{0}$ applies: ${ }^{4}$

$$
\begin{aligned}
A / R^{0}=2 & \pi^{-\frac{1}{2}}\left(S / P^{\prime}\right) z^{\frac{3}{2}} \\
& \times\left\{1-\left[2 \Gamma\left(\frac{1}{2}\right)\right]^{-1} \sum_{n=1}^{\infty}\left[\Gamma^{(n)}(1) \Gamma\left(n-\frac{1}{2}\right) /\left(n ! z^{n}\right)\right]\right\},
\end{aligned}
$$

where $z=\log \left(P^{\prime} X\right), \Gamma^{(n)}(1)$ is the $n$th derivative of the gamma-function evaluated at unit argument, and

$$
\Gamma(m)=\int_{0}^{\infty} e^{-t} t^{m-1} d t .
$$

2 For details see, for example, D. G. Kendall, Z. Astrophys. 16, 308 (1938).

${ }^{3}$ R. Ladenburg, Z. Physik 65, 200 (1930); R. D. Cowan and G. H. Dieke, Revs. Modern Phys. 20, 418 (1948).

${ }^{4}$ This expansion was obtained by H. S. Tsien. 
A nomogram for the determination of peak and total intensities for spectral lines with Doppler contour has been described previously. ${ }^{5}$ With the aid of this nomogram it is a simple matter to estimate intensities for temperatures between 1000 and $20000^{\circ} \mathrm{K}$, molecular weights between 2 and $80 \mathrm{~g} /$ mole, wavelengths between 0.10 and 0.80 micron, and values of $S X$ between $10^{-4}$ and $10 \mathrm{~cm}^{-1}$. The quantity $S$ is related to the $f$ value at the temperature $T$ through the expression

$$
S=2.3789 \times 10^{7}(273.1 / T) f .
$$

\section{B. Pure Collision Broadening}

The sum of the natural half-width $b_{N}$ and of the Lorentz half-width $b_{C}$ will be designated by the symbol $b$. In the absence of Doppler broadening it is well known that

and ${ }^{6,7}$

$$
P(\omega) \doteq(b / \pi)\left[\left(\omega-\omega_{0}\right)^{2}+b^{2}\right]^{-1} S
$$

$$
A / R^{0}=2 \pi b f(x) .
$$

The functions

$$
f(x)=x[\exp (-x)]\left[J_{0}(i x)-i J_{1}(i x)\right]
$$

have been tabulated by Elsasser, ${ }^{7}$ and

$$
x=S X / 2 \pi b \text {. }
$$

Here $J_{0}$ and $J_{1}$ are Bessel functions of order zero and one, respectively, and $i^{2}=-1$. Useful asymptotic forms $\mathrm{s}^{6,7}$ for $A / R^{0}$ are the following:

and

$$
A / R^{0} \sim S X \text { for small values of } x
$$

$$
A / R^{0} \sim 2(S b X)^{\frac{1}{2}} \text { for large values of } x,
$$

where Eq. (9a) is to be preferred for $x<(2 / \pi)$ and Eq. (9b) for $x>(2 / \pi)$.

Numerical values of $A$ for spectral lines with resonance contour can be obtained conveniently from a nomogram which is described elsewhere. ${ }^{8}$ This nomogram is useful for temperatures between 1000 and $20000^{\circ} \mathrm{K}$, values of $S X$ between $10^{-5}$ and $10^{+2} \mathrm{~cm}^{-1}$, half-widths $b_{N}+b_{C}$ between $10^{-2}$ and $1 \mathrm{~cm}^{-1}$, and wavelengths between 0.5 and 50 microns.

\section{EVALUATION OF $P(\omega)$ FOR COMBINED COLLISION AND DOPPLER BROADENING}

The calculation of $P(\omega)$ from Eq. (1) has been considered by a number of investigators. Before reviewing the various techniques which have been employed, we shall consider briefly several special cases.

Equation (1) can be transformed by a straightforward application of Parseval's theorem ${ }^{2}$ to a form first

\footnotetext{
5 Kavanagh, Björnerud, and Penner, J. Opt. Soc. Am. 43, 380 (1953).

${ }^{6}$ R. Ladenburg and F. Reiche, Ann. Physik 42, 181 (1913).

${ }^{7}$ W. M. Elsasser, Harvard Meteorological Studies No. 6, Milton (Massachusetts), 1942.

${ }^{8}$ R. W. Kavanagh and S. S. Penner, J. Opt. Soc. Am. 43, 483 (1953).
}

given by Reiche, ${ }^{9,10}$ viz.;

$$
P(\omega) / P^{\prime}=\pi^{-\frac{1}{2}} \int_{0}^{\infty}\left\{\exp \left[-a x-\left(x^{2} / 4\right)\right]\right\} \cos \xi x d x
$$

From Eq. (12) it is evident that the spectral absorption coefficient at the line center is always given by the expression

where

$$
P\left(\omega_{0}\right)=P^{\prime}\left[\exp \left(a^{2}\right)\right][\operatorname{erfc}(a)],
$$

$$
\operatorname{erfc}(a)=\left(2 / \pi^{\frac{1}{3}}\right) \int_{a}^{\infty}\left[\exp \left(-x^{2}\right)\right] d x=1-\operatorname{erf}(a)
$$

Born $^{11}$ has given an asymptotic expansion for $P(\omega) / P^{\prime}$ that is useful for $\xi / a \gg 1$. The complete expression is given in Eq. (18). For convenience the first two terms of the series are repeated here:

$$
P(\omega) / P^{\prime} \sim\left[a / \pi^{\frac{1}{2}}\left(a^{2}+\xi^{2}\right)\right]\left[1+\left(3 / 2 \xi^{2}\right)\right] .
$$

Born $^{11}$ has also derived an asymptotic expansion for $P(\omega) / P^{\prime}$ when $\xi / a \ll 1$. The complete expression is given in Eq. (19) and converges rapidly if $a$ is not too large. For $\xi=a$, Eq. (26) may be used to advantage.

Since $\xi / a$ must always become large in the wings of the lines, Eqs. (13) and (14) are convenient forms for determining the value of $P$ at the line center and its asymptotic limit in the wings of the line.

We now proceed to summarize briefly several special methods for the calculation of $P(\omega) / P^{\prime}$.

\section{A. $P(\omega) / P^{\prime}$ for Small Values of $a$}

Kendall has given a general expansion which can be used to determine $P(\omega) / P^{\prime}$ for small values of $a .^{2}$ His result is

$$
\begin{aligned}
P(\omega) / P^{\prime}=[ & \left.\exp \left(a^{2}\right)\right][\cos (2 a \xi)]\left[\exp \left(-\xi^{2}\right)\right] \\
& +2 \pi^{-\frac{1}{2}}\left[\exp \left(a^{2}\right)\right][\sin (2 a \xi)] F(\xi) \\
& -2 a \pi^{-\frac{1}{2}}\left[\exp \left(a^{2}\right)\right][\cos (2 a \xi)] \\
& \times \sum_{m=0}^{\infty}\left[a^{2 m} H_{2 m}(i \xi) /(2 m+1) !\right] \\
& +2 a \pi^{-\frac{1}{2}}\left[\exp \left(a^{2}\right)\right][i \sin (2 a \xi)] \\
& \times \sum_{m=0}^{\infty}\left[a^{2 m-1} H_{2 m-1}(i \xi) /(2 m) !\right]
\end{aligned}
$$

where $H_{n}(X)$ is the Hermite polynomial of degree $n$. The size of the remainder term can be estimated according to a procedure developed by E. C. Titchmarsh, which is described in Kendall's paper.

A useful expression for small values of $a$ is the

\footnotetext{
${ }^{9}$ F. Reiche, Verhandl. deut. physik. Ges. 15, 3 (1913).

${ }^{10}$ See also, A. Unsöld, Physik der Sternatmosphären (Edwards Brothers, Inc., Ann Arbor, 1948), pp. 159-169.

${ }^{11}$ M. Born, Optik (J. Springer, Berlin, 1933), pp. 482-486.
} 
Fig. 1. Extended curves of growth.

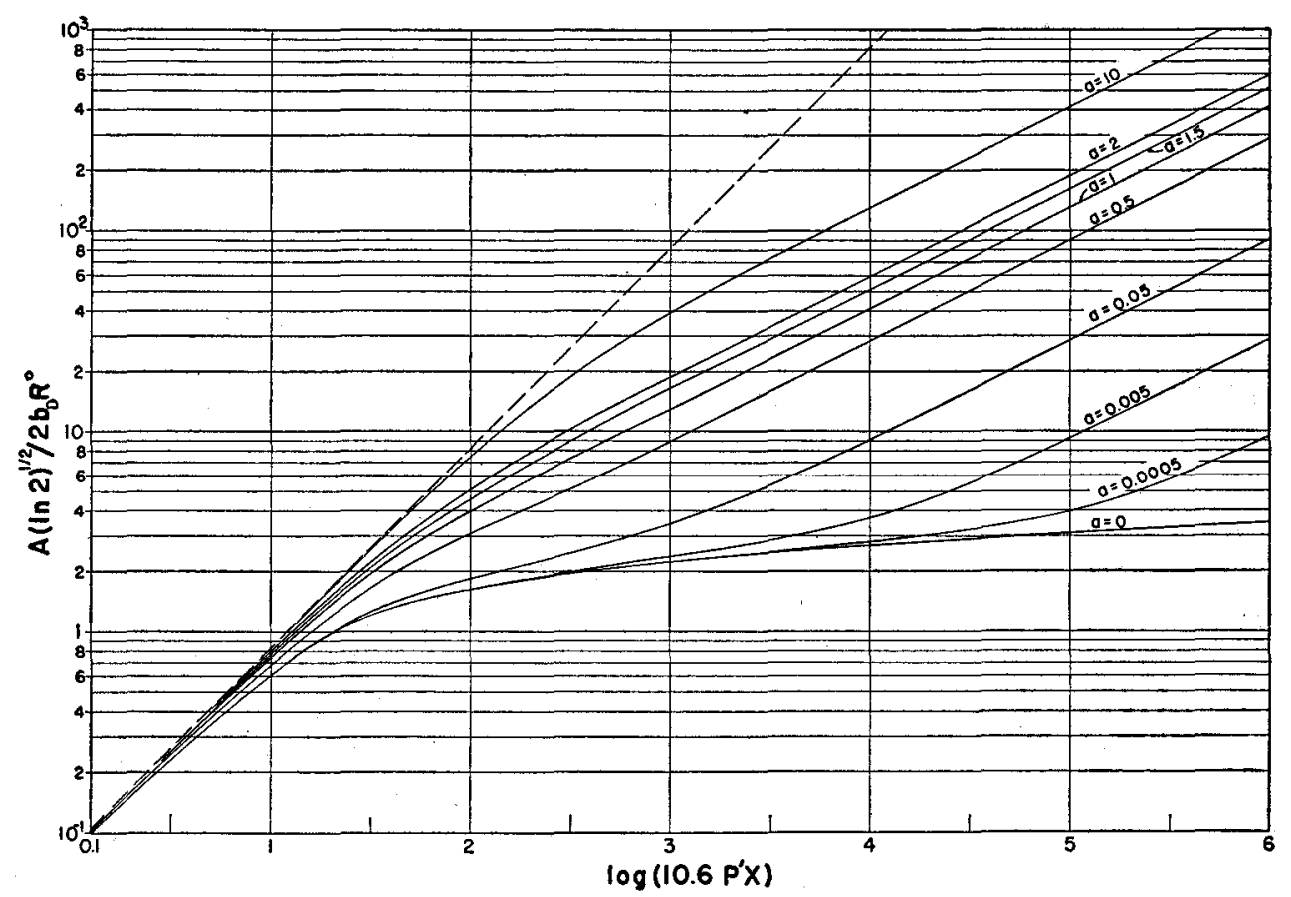

following:

$$
\begin{aligned}
P(\omega) / P^{\prime}= & \exp \left(-\xi^{2}\right)-2 a \pi^{-\frac{1}{2}}[1-2 \xi F(\xi)] \\
+ & a^{2}\left(1-2 \xi^{2}\right) \exp \left(-\xi^{2}\right)-2 a^{3} \pi^{-\frac{1}{2}}\left\{(2 / 3)\left(1-\xi^{2}\right)\right. \\
\left.-2 \xi\left[1-(2 / 3) \xi^{2}\right] F(\xi)\right\} & \\
& +a^{4}\left[\frac{1}{2}-2 \xi^{2}+(2 / 3) \xi^{4}\right] \exp \left(-\xi^{2}\right)+\cdots,
\end{aligned}
$$

where

$$
F(\xi)=\exp \left(-\xi^{2}\right) \int_{0}^{\xi}\left[\exp \left(x^{2}\right)\right] d x
$$

The first two terms of Eq. (16) were obtained by Mitchell and Zemansky, ${ }^{1}$ the next three are given by Kendall, ${ }^{2}$ and the entire expression is given by Harris. ${ }^{12}$ The functions $F(\xi)$ have been tabulated by Miller and Gordon..$^{13}$ Results which are accurate to 0.08 percent or better for $0 \leqslant a \leqslant 0.2$ are obtained by using the first three terms of Eq. (16). ${ }^{2}$ For $0 \leqslant a \leqslant 0.1$, the first two terms are sufficient. Numerical values of the coefficients of $a, a^{2}, a^{3}$, and $a^{4}$ in Eq. (16) have been tabulated by Harris ${ }^{12}$ as a function of $\xi$ for $0 \leqslant \xi \leqslant 12$. For larger values of $\xi$, i.e., large values of $\xi / a$, the asymptotic forms are suitable approximations.

\section{B. The Method of Born}

Born ${ }^{11}$ has obtained asymptotic expansions for $P(\omega)$ for large and for small values of $\xi / a$. For large $\xi / a$ it

12 D. L. Harris, III, Astrophys. J. 108, 112 (1948).

${ }^{13}$ W. L. Miller and A. R. Gordon, J. Phys. Chem. 35, 2877 1931). is shown that

$$
\begin{aligned}
P(\omega) / P^{\prime} & =\left(\frac{1}{a \sqrt{ } \pi} \frac{1}{1+(\xi / a)^{2}}\right) \\
\times & \left\{1-\frac{1}{2}\left(\frac{1}{a}\right)^{2} \frac{1-\left(\begin{array}{l}
3 \\
2
\end{array}\right)(\xi / a)^{2}}{\left[1+(\xi / a)^{2}\right]^{2}}+\frac{1 \times 3}{2^{2}}\left(\begin{array}{l}
1 \\
a
\end{array}\right)^{4}\right. \\
& \left.\times \frac{1-\left(\begin{array}{l}
5 \\
2
\end{array}\right)(\xi / a)^{2}+\left(\begin{array}{l}
5 \\
4
\end{array}\right)(\xi / a)^{4}}{\left[1+(\xi / a)^{2}\right]^{4}}+\cdots\right\} .
\end{aligned}
$$

On the other hand, for small values of $\xi / a$, Born gives the relation

$$
\begin{aligned}
& P(\omega) / P^{\prime}=\frac{2}{\sqrt{ } \pi} \exp \left(a^{2}-\xi^{2}\right) \\
& \times\left\{\operatorname { s i n } 2 a \xi \left\{\xi-\frac{a^{3}}{1 ! 3}\left[\left(\begin{array}{l}
3 \\
1
\end{array}\right)_{a}^{\xi}-\left(\begin{array}{l}
3 \\
3
\end{array}\right)\left(\frac{\xi}{a}\right)^{3}\right]\right.\right. \\
& \left.+\frac{a^{5}}{2 ! 5}\left[\left(\begin{array}{l}
5 \\
1
\end{array}\right)-\frac{\xi}{a}-\left(\begin{array}{l}
5 \\
3
\end{array}\right)\left(\frac{\xi}{a}\right)^{3}+\left(\begin{array}{l}
5 \\
5
\end{array}\right)\left(\frac{\xi}{a}\right)^{5}\right]+\cdots\right\} \\
& +\cos 2 a \xi\left\{\frac{\sqrt{ } \pi}{2}-a+\frac{a^{3}}{1 ! 3}\left[1-\left(\begin{array}{l}
3 \\
2
\end{array}\right)\left(\frac{\xi}{a}\right)^{2}\right]\right. \\
& \left.\left.-\frac{a^{5}}{2 ! 5}\left[1-\left(\begin{array}{l}
5 \\
2
\end{array}\right)\left(\frac{\xi}{a}\right)^{2}+\left(\begin{array}{l}
5 \\
4
\end{array}\right)\left(\frac{\xi}{a}\right)^{4}\right]+\cdots\right\}\right\}
\end{aligned}
$$


TABLE I. Comparison of notation used by different authors for equivalent physical quantities.

\begin{tabular}{cccc}
\hline \hline $\begin{array}{c}\text { Present } \\
\text { paper }\end{array}$ & Borna & Unsöld & $\begin{array}{c}\text { Mitchell and } \\
\text { Zemanskyo }\end{array}$ \\
\hline$\omega$ & $\omega / 2 \pi c$ & $\omega / 2 \pi c$ & $\tilde{\nu}=\nu / c$ \\
$P\left(\omega^{\prime}\right) / P^{\prime}$ & $\cdots$ & $k(\omega) / k_{0}, \omega=2 \pi c \omega^{\prime}$ & $k_{\nu} / k_{0}, \nu=c \omega^{\prime}$ \\
$b=b_{N}+b_{C}$ & $\gamma / 4 \pi c$ & $\gamma / 4 \pi c$ & $\frac{1}{2}\left(\Delta \nu_{N} / c\right)$ \\
$b_{D}$ & $(\delta / 4 \pi c)(\ln 2)^{\frac{1}{2}}$ & $(\ln 2)^{3} \Delta \omega_{D} / 2 \pi c$ & $\frac{1}{2}(\Delta \nu D / c)$ \\
$a$ & $1 / \eta$ & $a / 2$ & $a\left(\right.$ or $\left.a^{\prime}\right)$ \\
$\xi$ & $\left(\omega-\omega_{0}\right) / \frac{1}{2} \delta$ & $v$ & $\omega$ \\
$\xi / a$ & $x$ & $2 v / a$ & $\omega / a$ \\
\hline \hline
\end{tabular}

a See reference 11

See reference 10.

- See reference 1.

Equation (18) is most useful for values of $a$ larger than unity, whereas Eq. (19) converges rapidly for smaller values of $a$.

\section{The Method of Gronwall}

Gronwall ${ }^{14}$ transformed Eq. (1) to the form

$$
\begin{aligned}
P(\omega) / P^{\prime}=\left(2 / \pi^{\frac{1}{2}}\right) & {\left[\exp \left(a^{2}-\xi^{2}\right)\right] } \\
& \times \int_{a}^{\infty}\left\{\exp \left[-t^{2}+\left(a^{2} \xi^{2} / t^{2}\right)\right]\right\} d t .
\end{aligned}
$$

Expanding the term $\left[\exp \left(a^{2} \xi^{2} / t^{2}\right)\right]$ and integrating term by term, it is readily shown that

$$
P(\omega) / P^{\prime}=\sum_{n=0}^{\infty} I_{n}(a)(n !)^{-1}(\xi)^{2 n}\left[\exp \left(-\xi^{2}\right)\right]
$$

where

with

$$
I_{n}(a)=[a /(2 n-1)]\left[\left(2 / \pi^{\frac{1}{2}}\right)-2 a I_{n-1}(a)\right],
$$

$$
I_{0}(a)=\left[\exp \left(a^{2}\right)\right][\operatorname{erfc}(a)] .
$$

Equation (21) constitutes a useful approximation for relatively small values of $a$ and has been used by Mitchell and Zemansky ${ }^{1}$ to determine $P(\omega) / P^{\prime}$ for $a=0.5,1.0$, and 1.5 .

\section{Expression in Terms of Error Functions with Complex Arguments}

From Eq. (12) it can readily be shown ${ }^{2}$ that

$$
P(\omega) / P^{\prime}=R\left\{\left[\exp \left(z^{2}\right)\right][\operatorname{erfc}(z)]\right\},
$$

where $z=a+i \xi$ and $R$ denotes the real part of the following argument. Methods for calculating error functions with complex arguments, as well as numerical values, have been given by Salzer. ${ }^{15}$ From Eq. (24) it is readily shown that

$$
\begin{aligned}
& P(\omega) / P^{\prime}=\left[\exp \left(a^{2}-\xi^{2}\right)\right]\{[\cos (2 a \xi)][\operatorname{erfc}(a)] \\
&+(2 \pi a)^{-1}\left[\exp \left(-a^{2}\right)\right][1-\cos (2 a \xi)] \\
&-(2 / \pi)\left[\exp \left(-a^{2}\right)\right] \sum_{n=1}^{\infty}\left[\exp \left(-n^{2} / 4\right)\right]\left(n^{2}+4 a^{2}\right)^{-1} \\
&\quad \times\{2 a[\cos (2 a \xi)-\cosh (n \xi)]\}\}
\end{aligned}
$$

14 M. W. Zemansky, Phys. Rev. 36, 219 (1930).

${ }^{15}$ H. E. Salzer, "Mathematical Tables and Other Aids to Computation," National Research Council; April, 1951.
For $a=0$ Eq. (25) reduces to Eq. (4), which is the correct relation for pure Doppler broadening; at $\xi=0$, Eq. (13) results. The calculation of $P(\omega) / P^{\prime}$ from Eq. (25) is somewhat laborious for large values of $\xi$ and, therefore, asymptotic relations given previously are to be preferred in this case. For moderate or small values of $\xi$, Eq. (25) constitutes a useful expression for all values of $a$.

\section{E. Special Forms $\ddagger$}

For $\xi=a$ it can be shown that Eq. (25) reduces to the relation

$$
\begin{aligned}
P(\omega) / P^{\prime}= & {\left[\exp \left(a^{2}-\xi^{2}\right)\right] } \\
& \times\left\{\left[1-C\left(2 \pi^{-\frac{1}{2}} a\right)-S\left(2 \pi^{-\frac{1}{2}} a\right)\right] \cos \left(2 a^{2}\right)\right. \\
& \left.\quad+\left[C\left(2 \pi^{-\frac{1}{2}} a\right)-S\left(2 \pi^{-\frac{1}{2}} a\right)\right] \sin \left(2 a^{2}\right)\right\},
\end{aligned}
$$

where $C$ and $S$ are tabulated Fresnel integrals. ${ }^{15}$

For $a \gg 1$ and $\xi<a$ it can be shown that

$$
\begin{aligned}
P(\omega) / P^{\prime} & =\left(\pi^{\frac{1}{2}} a\right)^{-1} \sum_{n=0}^{\infty}(1 / 2)(3 / 2) \cdots[(2 n-1) / 2] a^{-2 n} \\
& \times\left(\sin ^{2 n+1} \theta\right)[\sin (2 n+1) \theta], \quad \pi / 4<\theta<\pi / 2,
\end{aligned}
$$

where $\theta=\tan ^{-1}(a / \xi)$.

\section{EXTENDED "CURVES OF GROWTH"}

The "curves of growth", which are familiar from astrophysical studies, ${ }^{16,17}$ constitute a useful method for the determination of the quantity $A / R^{0}$ defined by Eq. (3). Unfortunately the available curves do not cover the entire range of values of $a$ which is of interest in connection with spectroscopic studies on flames, particularly at atmospheric pressure. For this reason, calculations have been carried out to determine $A / R^{0}$ as a function of $X$ for $a=1.5,2$, and 10, using the numerical values for $P(\omega) / P^{\prime}$ given by Born ${ }^{11}$ and by Mitchell and Zemansky. ${ }^{1}$ The results of these calculations are shown in Fig. 1 together with van der Held's data. ${ }^{17}$ As was pointed out in a previous publication, ${ }^{5}$ absolute intensities for spectral lines with combined Doppler and collision broadening can be obtained by the combined use of a nomogram and of the curves of growth.

Since the notation used in the present investigations differs from that of other authors, a summary of notations for equivalent physical quantities is given in Table I.

$\ddagger$ The authors are indebted to Dr. H. S. Tsien for permission to reproduce the results given in this paragraph.

${ }^{15}$ See, for example, E. Jahnke and F. Emde, Tables of Functions with Formulae and Curves (Dover Publications, New York, 1943), pp. 34-35.

${ }^{16}$ Reference 1, p. 132; reference 10, p. 168.

${ }^{17}$ E. M. F. v. d. Held, Z. Physik 70, 508 (1931). 